\section{Mutations in hotspot region of MYH7 gene exon 23 associated with restrictive cardiomyopathy}

\author{
Mitali Kapoor, ${ }^{1}$ Soumi Das, ${ }^{1}$ \\ Amitabh Biswas, ${ }^{1}$ Sandeep Seth, ${ }^{2}$ \\ Balram Bhargava, ${ }^{2}$ \\ Vadlamudi Raghavendra Rao ${ }^{1,3,4}$
}

${ }^{1}$ Laboratory of Biochemical and

Molecular Anthropology, Department of Anthropology, University of Delhi, New

Delhi; ${ }^{2}$ Department of Cardiology, All

India Institute of Medical Sciences, New

Delhi; ${ }^{3}$ Department of Genetics,

Osmania University, Hyderabad;

${ }^{4}$ Genome Foundation, Hyderabad, India

\begin{abstract}
Restrictive cardiomyopathy (RCM) is characterized by restrictive filling of the ventricles. The association between the variable expressivity and age at onset of disease and disease complexity with double and compound heterozygous state is associated with severity of disease phenotype in recent reports. Sharing of variants of sarcomere genes across cardiomyopathies has implication in clinical expression of different clinical phenotypes. The present study reports Sanger DNA sequencing of $M Y H 7$ gene, exon 23 from 30 unrelated RCM patients and 15 primary relatives from sporadic families with the hypothesis that $\mathrm{RCM}$ has common etiology with hypertrophic cardiomyopathy (HCM). Rare variant E949K and a de novo compound heterozygous mutation (p.E902K and p.D906N), in two RCM patients with early onset and no ventricular hypertrophy were found. These variants wereabsent in 50 dilated cardiomyopathy, $50 \mathrm{HCM}$ patients and 15 primary relatives screened. The present report of rare and compound heterozygosity cases will further provide basis for the complexity and variable expressivity of phenotypes in patients in such complex diseases. The possible reasons for this phenotypic heterogeneity would be the presence of any other mutations in same chromosome or in different chromosome which is modifying the outcome of the causal mutation.
\end{abstract}

\section{Introduction}

Restrictive cardiomyopathy (RCM) is the least common type among cardiomyopathy disorders, which is defined as impaired ventricular filling, with normal or decreased diastolic volume of either or both ventricles. ${ }^{1}$ The rarity of this condition in childhood has made it very difficult to accurately assess outcomes, and risk factors for these outcomes, as well. ${ }^{2}$ With as many as $50 \%$ dying within 2 years of diagnosis, usually of sudden death, several studies have suggested an extremely poor outcome for this condition in childhood. ${ }^{3-9}$ The incidence of sudden cardiac death in pediatric patients with RCM, electrocardiographic evidence of ischemia has been reported as high as $23 \%{ }^{9}$

Primary cardiomyopathies were first linked to sarcomere genes when mutation in MYH7 and ACTC1 genes were reported to be associated with hypertrophic cardiomyopathy $(\mathrm{HCM})^{10}$ and dilated cardiomyopathy (DCM) ${ }^{11}$ Since then, till date more than 500 mutations were reported in HCM and DCM. In the last decade, restrictive cardiomyopathy was also found to be associated with mutations in sarcomeric genes, suggesting common etiologies among different cardiomyopathies. ${ }^{12}$ To date all sarcomeric proteins encoding genes are associated with the etiology of RCM with 33-66\% incident rate ${ }^{13,14}$ with TNNI3 being the first sarcomere gene implicated. However, there are many reports of $M Y H 7$ gene to be associated with RCM. ${ }^{15,16}$ MYH7 gene has a lot of hotspot region access based on the number of mutations found in the exon. Large number of studies reported mutations in exon 23 of $\mathrm{MYH7}$ associated with HCM proband and reported it as a hotspot region for the mutations involved in the etiology of cardiomyopathies. ${ }^{17-27}$

We report two idiopathic RCM patients with mutations in exon 23 of $M Y H 7$ gene in which one is rare and other two are novel compound heterozygote mutations. The report of these mutations will further increase knowledge about the genetic basis of restrictive cardiomyopathy and will help in expanding the pathophysiological pathways and therapeutic options.

\section{Materials and Methods}

\section{Clinical evaluation of patients}

Thirty patients diagnosed with restrictive cardiomyopathy between 2012 and 2015 by cardiologist at Out-patient Department (OPD), All India Institute of Medical Sciences (AIIMS), Delhi were enrolled for the study. Institutional ethical committee approved the study protocol and informed written consent was taken from all participants including family members. Familial case was considered when patient has any relative diagnosed with RCM and sporadic with unaffected relatives. When
Correspondence: Vadlamudi Raghavendra Rao, Laboratory of Biochemical and Molecular Anthropology (LBMA), Department of Anthropology, University of Delhi, New Delhi-110007, India.

Tel.: +01127667329 -

Fax: +01127666614

E-mail: profraoanthro@gmail.com

Key words: Restrictive cardiomyopathy; phenotypic heterogeneity; hypertrophic cardiomyopathy; genotypic heterogeneity; phenotype-genotype correlation.

Acknowledgments: we are thankful to al patients and their family members who have participated in the study and supportive staff at Cardiology Department, AIIMs for their cooperation.

Funding: the present study was conducted with the financial support from Department of Biotechnology (DBT), Govt. of India to VRR (BT/PR5767/MED/12/563/2012) and University Grant Commission (UGC) Senior Research Fellowship toMitali Kapoor (Schs/SRF/AA/139/F-185/2012-13)

Conflict of interest: the authors declare no potential conflict of interest.

Conference presentation: presented Poster entitled Clinical Characterization of Idiopathic Restrictive Cardiomyopathy having rare variant (E949K) in b-cardiac myosin heavy chain gene at annual conference of the Indian Society of Human Genetics held at Ahmadabad (22 $2^{\text {nd }}-25^{\text {th }}$ Dec, 2014).

Received for publication: 24 October 2016

Revision received: 18 March 2017.

Accepted for publication: 10 April 2017.

This work is licensed under a Creative Commons Attribution NonCommercial 4.0 License (CC BY-NC 4.0).

(C) Copyright M. Kapoor et al., 2017

Licensee PAGEPress, Italy

Cardiogenetics 2017; 7:6358

doi:10.4081/cardiogenetics.2017.6358

genetic variants were found in patients, screening in first-degree family members was done.

All patients and relatives underwentclinical examination, 12-lead electrocardiogram, 2D-echocardiography, cardiac catherisation and endomyocardial biopsy whenever required (Table 1). The diagnosis was based on echocardiographic demonstration of a bi-atrial enlargement, dilated inferior vena cava, restrictive doppler flows, relatively preserved systolic function, mildly reduced LV systolic function with markedly elevated ventricular filling 
pressures with characteristic restrictive hemodynamic pattern.

\section{Genetic analysis}

Sample preparations

Five milliliters of peripheral blood sample was collected in EDTA tubes. DNA was isolated using standardized phenol-chloroform method. ${ }^{28}$ The quantity and quality suitability for sequencing was checked by spectrophotometrically.

\section{DNA sequencing}

Appropriate primers for sequencing of the hotspot region of $M Y H 7$ gene i.e. exon 23 were used. ${ }^{17}$ The PCR product was sequenced by Sanger method (ABI 3730). Screening of available family members was also done.

\section{Results}

\section{Case \#1}

\section{Clinical assessment}

An 11-year old boy was diagnosed with primary RCM in the cardiology OPD at AIIMS, Delhi. The proband showed severe symptoms such as breathlessness on exertion, palpitation, giddiness, fatigability, pedal edema and chest discomfort for 6 months, aggravated for last 10 days. An electrocardiogram (ECG) disclosed sinus rhythm with marked right atrial enlarge- ment, left axis deviation, and nonspecific ST-T wave changes.

Jugular Venous pressure raised with prominent ' $Y$ ' descent. An echocardiography showed marked bi-atrial enlargement (LA=46 mm, $\mathrm{RA}=54 \mathrm{~mm}$ ), normal sized ventricles with wall thickness (IVS $=8 \mathrm{~mm}$, $\mathrm{LV}=9 \mathrm{~mm}$ ), mild mitral regurgitation (MR) and severe tricuspid regurgitation (TR). The left ventricular chamber size and wall thicknesses were normal (end diastolic dimension: $2.49 \mathrm{~cm}$ [reference range: 2.38-3.28 $\mathrm{cm}]$; end-diastolic wall thickness: $0.55 \mathrm{~cm}$ [reference range: $0.40-0.67 \mathrm{~cm}$ ]. Based on echocardiography and ECG findings a presumptive diagnosis of RCM was made. The proband was subsequently referred for cardiac catheterization. Cardiac catheterization revealed grade III MR, severe TR, severe $\mathrm{PAH}$, mild RV systolic dysfunction with left and right atrial waves measuring $27 \mathrm{mmHg}$ and $26 \mathrm{mmHg}$ respectively, no apical obliteration, no endocardial calcification and fair LV systolic dysfunction. The coronary artery anatomy and flow, as well as the pericardium, were normal. With these evidences, a diagnosis of idiopathic restrictive cardiomyopathy was confirmed. The age of presentation, first symptoms, and rapidity of the progress of the disease were consistent with a particularly severe form of RCM. The proband died due to sudden cardiac death after two years of diagnosis.

\section{Genetic analysis}

Genetic testing led to the identification of rare variant leading to the amino acid change, i.e. glutamic acid to lysine on position 949 (c.2845G>A) in MYH7 gene (Figure 1A). This variant was not found in our patient cohort of 29 other RCM and 15 primary relatives, $50 \mathrm{DCM}$ and $50 \mathrm{HCM}$ patients. Genetic and clinical screening was performed in ten available family members. All the family members were unaffected on clinical screening and the variant as well was not found.

\section{Case \#2}

\section{Clinical assessment}

A 24-year old lady was first listed with a prior complaint like palpitation, shortness of breath due to mild exertion, severe pedal edema and reduced urine output. On physical examination, peripheral edema was present and the jugular venous pressure was increased. On cardiac examination, a holosystolic murmur was present over the apex.

An ECG of a patient had heart rate 83 beats/min with normal sinus rhythm and left axis deviation. On echocardiography examination, the sizes of both ventricles were normal while atria were enlarged. IVC is dilated with $<50 \%$ variation with respiration. The left ventricle and right ventricle systolic function were found to be normal with $60 \%$ ejection fraction. There were no IVS thickening and no pericardial effusion

Table 1. Echocardiographic and ECG variables of patients.

\begin{tabular}{|c|c|c|c|c|c|}
\hline Age, sex & $\begin{array}{l}\text { Patient \#1 } \\
11 \text {, male }\end{array}$ & $\begin{array}{l}\text { Patient \#2 } \\
29 \text {, female }\end{array}$ & Age of onset (years) & $\begin{array}{c}\text { Patient \#1 } \\
9\end{array}$ & $\begin{array}{l}\text { Patient \#2 } \\
24\end{array}$ \\
\hline \multicolumn{4}{|c|}{ Echocardiography variables } & \multicolumn{2}{|c|}{ ECG variables } \\
\hline Aorta $(21-22$ mm/m²) & 21 & 27 & ST-T changes & Present & Present \\
\hline $\begin{array}{l}\text { Lves } \\
\left(16-19 \mathrm{~mm} / \mathrm{m}^{2}\right)\end{array}$ & 23 & 28 & Tachycardia & Absent & Present \\
\hline $\begin{array}{l}\text { IVSed } \\
(6-10 \mathrm{~mm})\end{array}$ & 8 & 11 & Brachycardia & Absent & Absent \\
\hline LAes $\left(21-22 \mathrm{~mm} / \mathrm{m}^{2}\right)$ & 49 & 47 & Left axis deviation & Absent & Present \\
\hline $\begin{array}{l}\text { LVed } \\
\left(19-32 \mathrm{~mm} / \mathrm{m}^{2}\right)\end{array}$ & 33 & 45 & Sinus rhythm & Present & Present \\
\hline $\begin{array}{l}\text { LVEF } \\
(62-80 \%)\end{array}$ & $43 \%$ & $63 \%$ & Atrial fibrillation & Present & Absent \\
\hline Mitral Regurgitation & Mild & Mild & LBBB & Absent & Present \\
\hline Tricuspid Regurgitation & Severe & Severe & RBBB & Absent & Present \\
\hline
\end{tabular}

Table 2. In silico analysis of both compound heterozygous mutations in RCM proband.

\begin{tabular}{lccc} 
Variants & Polyphen-2 & Sift & Panther \\
E949K & Probably damaging & Tolerated & Deleterious effect \\
E902K & Pathogenic & Damaging & Deleterious effect \\
\hline D906N & Benign & Probably Damaging & Deleterious effect \\
\hline
\end{tabular}


present. Left ventricular diastolic function was abnormal with $E^{\prime}<A^{\prime}, E / E^{\prime} \sim 11$ with fraction shortening $30-41 \%$. Doppler study showed mild MR, severe TR and pulmonary stenosis with peak gradient 36 $\mathrm{mmHg}$. On cardiac catherisation examination, the elevation in both side pressures, with a prominent y-descent suggesting a dip and plateau and ventricular concordance was present.

\section{Genetic analysis}

In genetic analysis, Sanger sequencing led to the identification of compound heterozygous mutations E902K and D906N in close proximity and on genetic testing of parents these mutations were found to be de novo (Figure1B).

\section{Discussion}

The importance of genetic testing in the etiologies of cardiomyopathies has been recognized over the last decade. RCM constitute nearly $5 \%$ of total pediatric cases among different types of cardiomyopathies..$^{29}$ Due to progression in molecular techniques, mutations in all eight sarcomeric genes are now recognized to be linked with RCM, but the pathophysiological mechanism leading to the final disease phenotype is still unknown. The two utmost implicated genes in this rare cardiomyopathy are TNNI3 and MYH7 genes. However, MYH7 gene has been mostly linked with HCM rather than RCM. Here we report one rare $(\mathrm{E} 949 \mathrm{~K})$ and novelcompound heterozygous mutations (E902K and D906N) in exon 23 of $\beta$-myosin heavy chain (MYH7). The Exome Aggregation Consortium (EXac) database was searched for these rare and compound heterozygote variant and found $\mathrm{E} 949 \mathrm{~K}$ is rare variant reported by only one HCM family ${ }^{30}$ with MAF of 0.000008236 and the compound heterozygous variants (E902K and D906N) to be novel variants. ${ }^{31}$

The p.E949K mutation is a rare variant found in RCM patient represents an early age of onset and no ventricular hypertrophy, while by reports, it was implicated with familial HCM suggesting late onset of disease with severe left ventricular hypertrophy earlier reported in patient with European descent, ${ }^{30}$ where as present patients are of south Asian, north Indian ethnicity. The important fact is that, the mutation found is located in the extremely conserved region. The mutation in $M Y H 7$ is recognized as origin of severe clinical outcomes and early age penetrance with a high frequency of sudden cardiac death ${ }^{32}$ which is clearly seen in this patient having a severe form of RCM and early age of onset followed by sudden cardiac death. This mutation has occurred in $\alpha$ - helical segment that connects the myosin head to the backbone of the thick filament. This could affect assembly of the thick filament or stability of protein; the effect may be due to a loss of tensile strength or stiffness and may result in disease phenotype..$^{33}$ The phenotypic heterogeneity in two patients having the same pathogenic mutation present in two different cardiomyopathies from two different ethnic backgrounds. The other possible reasons for this complexity of the phenotypic heterogeneity would be the presence of any other mutations in same chromosome or in different chromosome or possibility of multiple interactions between genes, or and other modifying factors in the outcome of disease condition.

Compound heterozygote mutations are now frequently seen to be associated with other cardiomyopathies i.e. dilated and hypertrophic cardiomyopathy. ${ }^{34}$ HCM shows $4 \%$ of cases with two mutations. ${ }^{35,36}$ Recently, many studies also reported cases with RCM associated with compound het- erozygous and double heterozygous mutations. ${ }^{34,37}$ A 9-year old girl with RCM has been found to have two mutations in TNNI3 gene, i.e. compound heterozygous while Peddy et al. reported double heterozygous mutation in an infant RCM case showing an early age of onset. ${ }^{34,37}$ Two or more than two mutations in the same gene or different gene are known to cause early age of onset and the severe clinical phenotypes. Patient 2 with compound heterozygous mutation also presented with an early age of onset with severe symptoms leading to NYHA-IV class from II in the span of 1 year and is now a potential case for heart transplant. These both mutations are present on the rod like structure of $M Y H 7$ gene and are conserved in all mammalian species, i.e. likely to be pathogenic as they are present on important domain of the protein.

In silico assessment of these rare variants through different bioinformatics tools predicted to have a deleterious effect (Table 2). The causality of the $M Y H 7$ p.E949K, p.E902K and p.D906N mutations in the case of RCM is pathogenic since this mutation was observed neither in unaffected
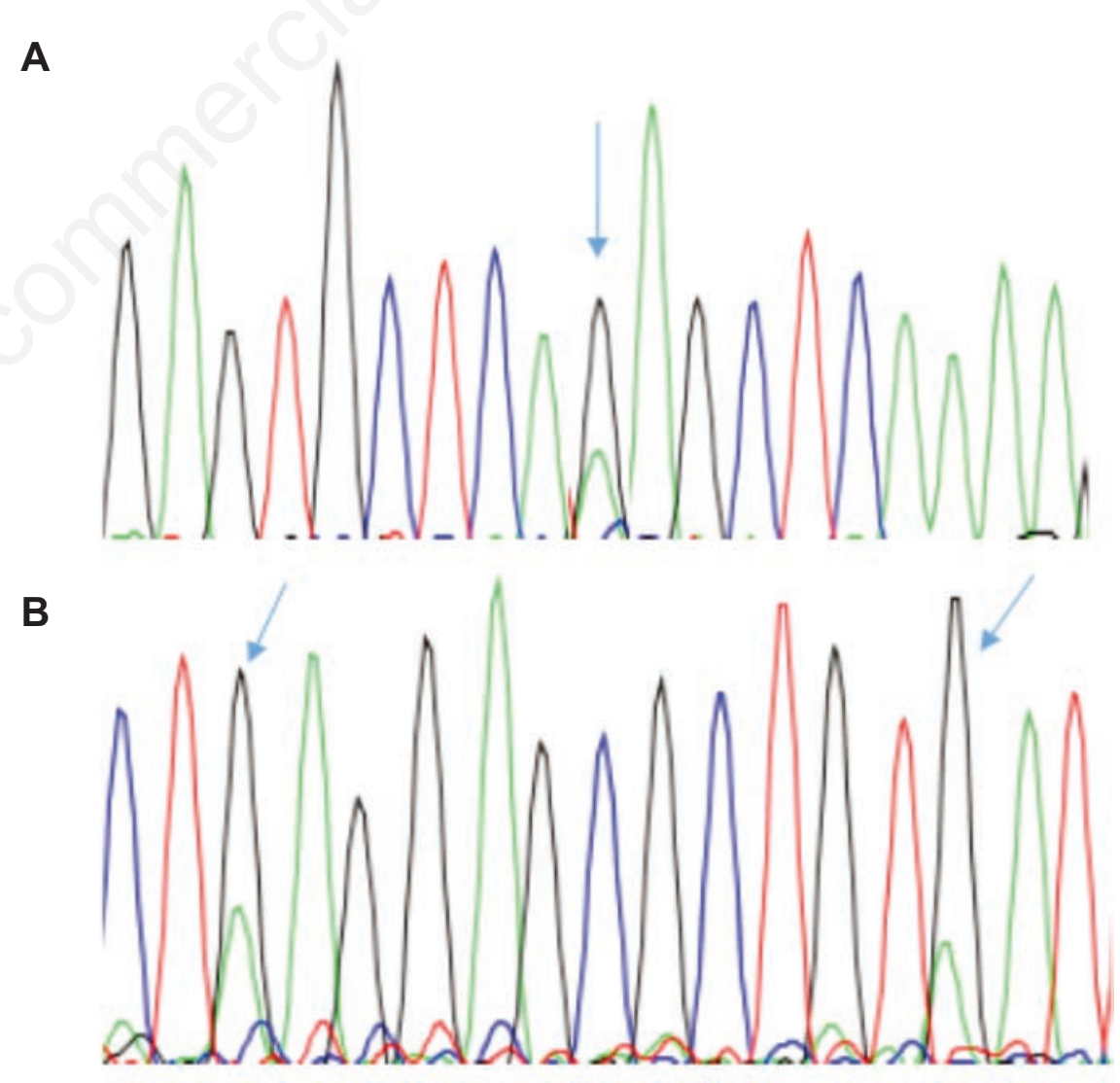

Figure 1. A) Shows the sequence of exon 23 of MYH7 gene with an arrow pointing to rare $\mathrm{G}$ to A changes at codon 949. B) Shows a compound heterozygous variants. Arrows point to the identified alterations $\mathrm{G}$ to A changes at codon 902 and 906. 
family members nor in in-house tested 130 cardiomyopathies patients. The limitation of the study is that we could not undertake sequencing of other common sarcomere genes.

\section{Conclusions}

This study reiterates common etiology for RCM and HCM and represents the phenotypic heterogeneity of same rare genetic variant in two ethnically different patients. This also provides basis that expression of disease phenotype may be modified by different mutations in same gene or different modifying factors. From this study, it is advisable to screen all the candidate genes/whole exome to explain the molecular mechanism which leads to possible phenotypic outcomes, and the advent of next generation sequencing (NGS) technologies make it a possible approach.

\section{References}

1. Maron BJ, Towbin JA, Thiene G, et al. Contemporary definitions and classification of the cardiomyopathies. Circulation 2006;113:1807-16.

2. Webber SA, Lipshultz SE, Sleeper LA, et al. Outcomes of restrictive cardiomyopathy in childhood and the influence of phenotype: a report from the Pediatric Cardiomyopathy Registry. Circulation 2012;126:1237-44.

3. Lewis AB. Clinical profile and outcome of restrictive cardiomyopathy in children. Am. Heart J 1992;123:1589-93.

4. Cetta F, O'Leary PW, Seward JB, et al. Idiopathic restrictive cardiomyopathy in childhood: diagnostic features and clinical course. Mayo Clin Proc 1995;70:634-40.

5. Denfield SW, Rosenthal G, Gajarski RJ, et al. Restrictive cardiomyopathies in childhood. Etiologies and natural history. Tex Heart Inst J 1997;24:38.

6. Chen SC, Balfour IC, Jureidini S. Clinical spectrum of restrictive cardiomyopathy in children. J Heart Lung Transplant 2001;20:90-2.

7. Weller RJ, Weintraub R, Addonizio LJ, et al. Outcome of idiopathic restrictive cardiomyopathy in children. Am J Cardiol 2002;90:501-6.

8. Russo LM, Webber SA. Idiopathic restrictive cardiomyopathy in children. Heart 2005;91:1199-202.

9. Rivenes SM, Kearney DL, Smith EB, et al. Sudden death and cardiovascular collapse in children with restrictive cardiomyopathy.

Circulation 2000;102:876-82.

10. Geisterfer-Lowrance AA, Kass S, Tanigawa $\mathrm{G}$, et al. A molecular basis for familial hypertrophic cardiomyopathy: a $\beta$ cardiac myosin heavy chain gene missense mutation. Cell 1990;62:9991006.

11. Olson TM, Michels VV, Thibodeau SN, et al. Actin mutations in dilated cardiomyopathy, a heritable form of heart failure. Science 1998;280:750-2.

12. Perrot A, Dietz R, Osterziel KJ. Is there a common genetic basis for all familial cardiomyopathies?. Eur J Heart Fail 2007;9:4-6.

13. Bahl A, Saikia UN, Khullar M. Idiopathic restrictive cardiomyopathyperspectives from genetics studies. Is it time to redefine these disorders? Cardiogenetics 2012;2:e4.

14. Caleshu C, Sakhuja R, Nussbaum RL, et al. Furthering the link between the sarcomere and primary cardiomyopathies: restrictive cardiomyopathy associated with multiple mutations in genes previously associated with hypertrophic or dilated cardiomyopathy. Am J Med Genet Part A 2011;155:2229-35.

15. Kubo T, Kitaoka H, Okawa M, et al. Lifelong left ventricular remodeling of hypertrophic cardiomyopathy caused by a founder frameshift deletion mutation in the cardiac myosin-binding protein $\mathrm{C}$ gene among Japanese. J Am Coll Cardiol 2005;46:1737-43.

16. Greenway SC, Wilson GJ, Wilson J, et al. Sudden death in an infant with angina, restrictive cardiomyopathy, and coronary artery bridging an unusual phenotype for a $\beta$-myosin heavy chain (MYH7) sarcomeric protein mutation. Circ Heart Fail 2012;5:e92-3.

17. Van Driest SL, Jaeger MA, Ommen SR, et al. Comprehensive analysis of the beta-myosin heavy chain gene in 389 unrelated patients with hypertrophic cardiomyopathy. J Am Coll Cardiol 2004;44:602-10.

18. Yu B, Sawyer NA, Caramins M, et al. Denaturing high performance liquid chromatography: high throughput mutation screening in familial hypertrophic cardiomyopathy and SNP genotyping in motor neurone disease. J Clin Pathol 2005;58:479-85.

19. Perrot A, Schmidt-Traub H, Hoffmann B, et al. Prevalence of cardiac betamyosin heavy chain gene mutations in patients with hypertrophic cardiomyopathy. J Mol Med 2005;83:468-77.

20. Erdmann J, Daehmlow S, Wischke S, et al. Mutation spectrum in a large cohort of unrelated consecutive patients with hypertrophic cardiomyopathy. Clin Genet 2003;64:339-49.

21. Ho CY, Sweitzer NK, McDonough B, et al. Assessment of diastolic function with Doppler tissue imaging to predict genotype in preclinical hypertrophic cardiomyopathy. Circulation 2002;105: 2992-7

22. Mohiddin SA, Begley DA, McLam E, et al. Utility of genetic screening in hypertrophic cardiomyopathy: prevalence and significance of novel and double (homozygous and heterozygous) $\beta$-myosin mutations. Genet Test 2003;7: 21-7.

23. Woo A, Rakowski H, Liew JC, et al. Mutations of the $\beta$ myosin heavy chain gene in hypertrophic cardiomyopathy: critical functional sites determine prognosis. Heart 2003;89:1179-85.

24. Song L, Zou Y, Wang J, et al. Mutations profile in Chinese patients with hypertrophic cardiomyopathy. Clin Chim Acta 2005;351:209-16.

25. Mörner S, Richard P, Kazzam E, et al. Identification of the genotypes causing hypertrophic cardiomyopathy in northern Sweden. J Mol Cell Cardiol 2003;35:841-9.

26. Waldmüller S, Sakthivel S, Saadi AV, et al. Novel deletions in MYH7 and MYBPC3 identified in Indian families with familial hypertrophic cardiomyopathy. J Mol Cell Cardiol 2003;35: 623-36.

27. Morita H, Rehm HL, Menesses A, et al. Shared genetic causes of cardiac hypertrophy in children and adults. N Engl J Med 2008;358:1899-908.

28. Sambrook J, Fritsch EF, Maniatis T. Molecular cloning: a laboratory manual, 2nd ed. New York: Cold Spring Harbor Laboratory Press; 1989.

29. Karam S, Raboisson MJ, Ducreux C, et al. A de novo mutation of the beta cardiac myosin heavy chain gene in an infantile restrictive cardiomyopathy. Congenit Heart Dis 2008;3:138-43.

30. Watkins H, Rosenzweig A, Hwang DS, et al. Characteristics and prognostic implications of myosin missense mutations in familial hypertrophic cardiomyopathy. $\mathrm{N}$ Engl J Med 1992;326:1108-14.

31. Exome Aggregation Consortium. ExAC Browser (Beta). Availabe from: http://exac.broadinstitute.org

32. Charron P, Dubourg O, Desnos M, et al. Diagnostic value of electrocardiography and echocardiography for familial hypertrophic cardiomyopathy in a genotyped adult population. Circulation 1997;96:214-9.

33. Rayment I, Holden HM, Sellers JR, et 
al. Structural interpretation of the mutations in the beta-cardiac myosin that have been implicated in familial hypertrophic cardiomyopathy. Proc Natl Acad Sci U S A 1995;92:3864-8.

34. Blok R, van den Wijngaard A, Merckx $\mathrm{D}$, et al. Two novel TNNI3 mutations in restrictive cardiomyopathy [poster P0135]. Poster presented at: European Human Genetics Conference, May 710, 2005, Prague, Czech Republic. Available from: www.eshg.org/ eshg2005/index1.htm Accessed: March 15, 2005. 35. Van Driest SL, Ommen
SR, Tajik AJ, et al. Sarcomeric genotyping in hypertrophic cardiomyopathy. Mayo Clin Proc 2005;80:463-9.

36. Kelly M, Semsarian C. Multiple mutations in genetic cardiovascular disease. A marker of disease severity? Circ Cardiovasc Genet 2009;2:182-90. 